Bundesgesundheitsbl -

Gesundheitsforsch - Gesundheitsschutz

2000 [Suppl 1] 43:S3-S8 @ Springer-Verlag 2000

Workshop HIV-Postexpositionsprophylaxe

U. Marcus $\cdot 0$. Hamouda

Robert Koch-Institut, Berlin

\title{
Epidemiologie der HIV-Neuinfektion in den verschiedenen Risikogruppen
}

\section{Zusammenfassung}

Die HIV-Epidemie in Deutschland kann bis zu den frühen neunziger Jahren am besten mittels Back-calculation-Modellen beschrieben werden. Solche von den bereits diagnostizierten AIDS-Fällen aus rückrechnenden Modelle können prinzipiell die aktuellere Entwicklung der HIV-Epidemie der letzten drei bis vier Jahre nicht gut erfassen. Darüber hinaus verliert diese Methodik durch die stärkeren Einflüsse der Behandlung der HIVInfektion auf den natürlichen Infektionsverlauf an Aussagekraft.Zur Beschreibung des aktuellen Verlaufes der HIV-Epidemie muss man sich mangels anderer Datenquellen auf die Meldungen von HIV-Diagnosen stützen. Danach dürfte die Zahl der jährlichen HIVNeuinfektionen in Deutschland seit einigen Jahren relativ stabil zwischen 2000 und 2500 pro Jahr liegt.Größte Betroffenengruppe sind noch immer homo- und bisexuelle Männer, i.v.-Drogengebraucher sind von Migranten aus Hochprävalenzregionen und heterosexuell infizierten Personen auf den zahlenmäßig vierten Rang verdrängt worden. Eine Zunahme von Risikoverhalten und HIV-Neuinfektionen kann derzeit in Deutschland weder bestätigt, noch mit Sicherheit ausgeschlossen werden. Zur frühzeitigen Erfassung einer Änderung von Risikoverhalten wäre eine Verbesserung der epidemiologischen Erfassung von STDs mit kurzen Inkubationszeiten wünschenswert.

\section{Schlüsselwörter}

HIV-Epidemie · HIV-Neuinfektionen · Backcalculation - epidemiologische Erfassung
Wer Aussagen über den Verlauf der HIV-Epidemie in Deutschland machen will, muss berücksichtigen, dass diese Epidemie nur schwer direkt beobachtet werden kann. Zum einen wurde die Epidemie erst mit der Entwicklung von Antikörper-Nachweisverfahren (ab 1984/85) messbar, und zu diesem Zeitpunkt waren bereits tausende von Personen infiziert, zum anderen wird die HIV-Infektion eher selten unmittelbar nach der Übertragung diagnostiziert. Zwar erkrankt die Mehrheit der Infizierten an einem sogenannten akuten retroviralen Syndrom mit Fieber, Lymphknotenschwellungen, einem flüchtigen Exanthem und anderen unspezifischen Symptomen (s. Tabelle 1 [1]), aber dieses Krankheitsbild ist oft nur schwach ausgeprägt und veranlasst daher nicht $\mathrm{zu}$ einem Arztbesuch bzw. wird vom Arzt, insbesondere wenn keine genaue Risikoanamnese erhoben wird, differentialdiagnostisch nicht berücksichtigt.

\section{Back-calculation-Modelle}

Aus dem Verlauf der AIDS-Epidemie, d.h. der manifesten AIDS-Erkrankungen, und mit Hilfe der aus Kohortenstudien gewonnenen Informationen zum natürlichen Verlauf (d.h. ohne Berücksichtigung von Behandlungseffekten) lässt sich der bisherige Ablauf der HIVEpidemie in Deutschland relativ gut mathematisch modellieren. Aus der Anzahl der aufgetretenen AIDS-Fälle (bis zum Wirksamwerden schwer modellierbarer
Therapieeffekte ca. 1996) und der Kenntnis der Inkubationszeitverteilung von der HIV-Infektion bis zum AIDS-Vollbild lässt sich (Abb. 1) mit Hilfe solcher Back-calculation-Modelle der Ablauf der HIV-Epidemie rekonstruieren (Abb. 2). Naturgemäß können aber über Back-calcution keine Aussagen über jüngere Entwicklungen (d.h. der letzten drei bis vier Jahre) gemacht werden, und außerdem nimmt die ,Repräsentativität” des AIDS-Fallregisters für die HIVEpidemie seit ca. 1996 deutlich ab, weil durch die seit dieser Zeit eingesetzten effektiveren Therapien das AIDS-Fallregister nicht mehr allein die HIV-Epidemie mit einigen Jahren Verzögerung widerspiegelt, sondern zunehmend auch Aspekte wie den Zugang zu und die Wirksamkeit von Therapien, die bis 1995 für den Ablauf der AIDS-Epidemie noch keine große Rolle spielten.

Für den zeitlichen Verlauf der HIVInzidenz in den verschiedenen Risikogruppen ergibt sich nach unseren heutigen Kenntnissen und nach den Ergebnissen des oben beschriebenen Rückrechnungsmodells folgendes Bild.

\section{Homo- bzw. bisexuelle Männer}

Nachdem sich die HIV-Epidemie -wahrscheinlich Ende der 7oer Jahre -unter homo- bzw. bisexuellen Männern in der

Dr.Ulrich Marcus

Dr. Osmah Hamouda, Robert Koch-Institut, Nordufer 20,13353 Berlin 
Bundesgesundheitsbl -

Gesundheitsforsch - Gesundheitsschutz

2000 [Suppl1] 43: S3-S8 ๔ Springer-Verlag 2000

\section{U.Marcus $\cdot 0$. Hamouda}

\section{Epidemiology of HIV infections in} different groups at risk in Germany

\section{Summary}

The course of the german HIV epidemic can be modeled reliably by back calculation until the beginning of the nineties. The actual course of the epidemic can only be monitored by the epidemiological surveillance of newly diagnosed HIV infections. Based on these surveillance data HIV incidence in Germany can be estimated to be between 2000 and 3000 new infections per year. The most affected group are still homo- and bisexual men.IDU - during the eighties the second largest group - have been surpassed meanwhile by migrants from high prevalence regions and by heterosexually infected persons. Increases of risk behavior and of new HIV infections as described in some studies from the US can neither be confirmed nor be refuted in Germany due to the lack of reliable data. An improvement of the epidemiological surveillance for "indicator" STDs would be desirable.

\section{Key words}

HIV epidemic · HIV incidence · Back calculation · Epidemiological surveillance

\section{Workshop HIV-Postexpositionsprophylaxe}

Tabelle 1

Klinische Symptomatik bei akuter HIV-Infektion

Symptomatik

Häufigkeit in Prozent*

$\begin{array}{ll}\text { Fieber }\left(>38^{\circ} \mathrm{C}\right) & 77 \\ \text { Abgeschlagenheit } & 66 \\ \text { Hautexanthem } & 56 \\ \text { Muskelschmerzen } & 55 \\ \text { Kopfschmerzen } & 51 \\ \text { Pharyngitis (Rachenentzündung) } & 44 \\ \text { Zervikale Lymphadenopathie } & 39 \\ \text { Gelenkschmerzen } & 31 \\ \text { Orale Ulzera } & 29 \\ \text { Axilläre Lymphadenopathie } & 24 \\ \text { Gewichtsverlust } & 24 \\ \text { Übelkeit } & 24 \\ \text { Durchfall } & 23 \\ \text { Nachtschweiß } & 22 \\ \text { Husten } & 22 \\ \text { Appetitlosigkeit } & 21 \\ \text { Inguinale Lymphadenopathie } & 20 \\ \text { Bauchschmerzen } & 19 \\ \text { Orale Candidose } & 17\end{array}$

*Grundlage sind die klinischen Befunde bei 218 Personen, bei denen eine akute HIV-Infektion klinisch und serologisch diagnostiziert wurde

Bundesrepublik Deutschland auszubreiten begann, erreichte die Inzidenz in dieser Gruppe mit etwa 5000 Neuinfektionen pro Jahr bereits um 1983 ihren Höhepunkt. Es muss davon ausgegangen werden, dass es gegenwärtig trotz aller Präventionsbemühungen unter homo- bzw. bisexuellen Männern noch zu etwa 1200 bis 1500 Neuinfektionen pro Jahr kommt.

Diese Gruppe ist es, die zur Zeit am meisten von den verbesserten therapeutischen Möglichkeiten profitiert. Ihr relativer Anteil an der Gesamtzahl der pro Jahr diagnostizierten AIDS-Fälle hat sich - nicht zuletzt deswegen - von $72 \%$ für die Jahre vor 1988 auf $49 \%$ im Jahr 1998 verringert.

\section{I.v.-Drogenabhängige}

Die HIV-Epidemie begann bei den durch i.v.-Drogenkonsum Infizierten etwas später als bei homo- und bisexuellen Männern. Der Höhepunkt der HIVInzidenz in dieser Gruppe wurde wahrscheinlich 1984 mit ungefähr $1500 \mathrm{Neu}$ infektionen pro Jahr erreicht. Gegenwärtig muss man davon ausgehen, dass sich etwa 300 bis 400 i.v.-Drogenkonsumen- ten pro Jahr neu mit HIV infizieren. Verhaltensänderungen haben bei den i.v.Drogenabhängigen -stärker als zu Beginn der Epidemie erwartet - stattgefunden. Während die Wiederverwendung bzw. der gemeinsame Gebrauch von unzureichend gereinigten Spritzen und Nadeln im allgemeinen deutlich eingeschränkt wurde, bleibt deren mehrfache Benutzung von verschiedenen Personen an Orten, wo saubere Spritzbestecke nicht uneingeschränkt verfügbar sind (u.a. in Haftanstalten), eine erhebliche Infektionsgefahr.

\section{Heterosexuell Infizierte}

In der Gruppe der über heterosexuelle Kontakte Infizierten begann die HIVEpidemie gegenüber den anderen Risikogruppen mit einer deutlichen Verzögerung, da es sich in erster Linie um sekundäre Infektionen über Partner aus anderen - primär betroffenen - Gruppen handelt. Zur Zeit muss in dieser Gruppe mit etwa 300 bis 400 Neuinfektionen pro Jahr gerechnet werden. Auch wenn es keine Hinweise dafür gibt, dass HIV sich außerhalb der sogenannten primären Risikogruppen eigenständig 
und massiv in der Bevölkerung ausbreitet - vergleichbar etwa der Ausbreitung von HIV in der ersten Hälfte der 8oer Jahre unter promisk lebenden homosexuellen Männern - ist in dieser Risikogruppe in erster Linie durch Sekundärinfektionen von Partnern aus den hauptbetroffenen Gruppen auch in den nächsten Jahren noch mit einer Steigerung zu rechnen.

Eine eigenständige Epidemie innerhalb der allgemeinen heterosexuellen Bevölkerung hat sich bisher nicht entwickelt und ist nach heutiger Einschätzung in der näheren Zukunft auch nicht zu erwarten. Rahmenbedingungen und Risikofaktoren für eine heterosexuelle Epidemie (u.a. hohe Prävalenz anderer sexuell übertragbarer Krankheiten, geringe Kondomverwendungsrate im Prostitutionsbereich) sind vergleichsweise gering ausgeprägt. Jugendliche, bei denen es vergleichsweise häufig zum Partnerwechsel kommt und die eine relativ hohe Inzidenz für virale und bakterielle sexuell übertragbare Infektionen aufweisen können, sind in den meisten Industriestaaten durch Alterskohorteneffekte von der HIV-Epidemie abgeschirmt (der Schwerpunkt der Epidemie liegt in den Industriestaaten im Unterschied zu den meisten Entwicklungsländern in den Altersgruppen der 25- bis 35Jährigen). Die Anzahl der potentiell infektiösen Kontakte (Anzahl der Sexualpartner, Anzahl der Kontakte pro Partner), die eine infizierte heterosexuelle Person innerhalb der Zeiten hoher Infektiosität hat, reichen in Deutschland daher anscheinend nicht aus, um den „kritischen” Wert für eine eigenständige Reproduktion der HIV-Epidemie zu übersteigen. Die bisher beobachteten Infektionsketten brechen nach wenigen „Gliedern” ab. Die Einschätzung, dass die Verbreitung der HIV-Infektion in der allgemeinen heterosexuellen Bevölkerung bisher kein großes Ausmaß angenommen hat, wird auch durch die Ergebnisse des Anonymen Unverknüpften Testens (AUT) bei Neugeborenen und den Untersuchungen von Blutspendern bestätigt. Dies schließt jedoch nicht aus, dass HIV, wenn es in entsprechende Subpopulationen eingeführt wird, sich dort auch auf heterosexuellem Wege endemisch verbreiten kann. Die weitere Entwicklung der HIV-Epidemie bei Personen mit heterosexuellem Infektionsrisiko wird maßgeblich davon abhängen, ob

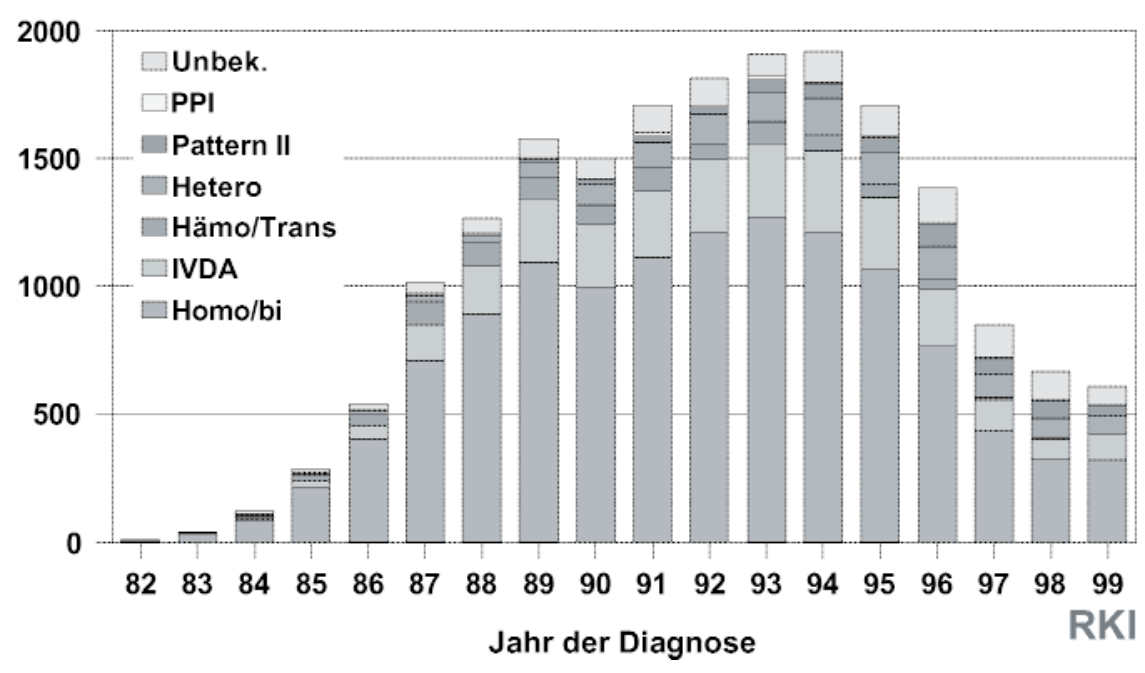

Abb. $1 \Delta$ Anzahl der jährlichen AIDS-Diagnosen in Deutschland, nach Infektionsrisiko korrigiert um den Meldeverzug

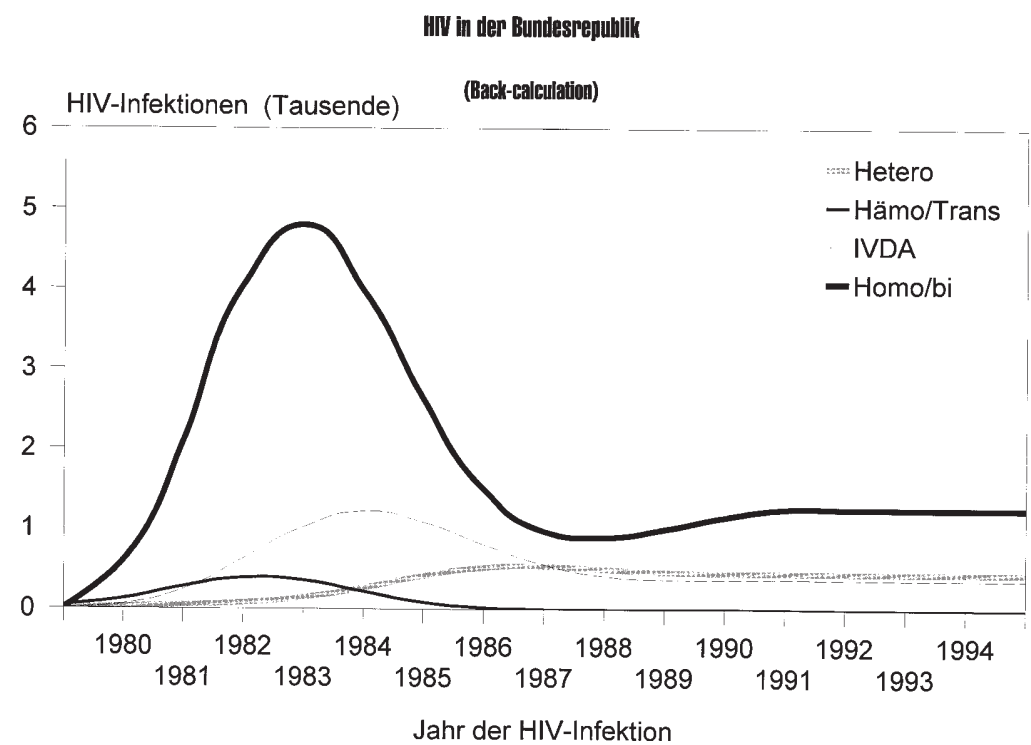

Abb. $2 \Delta$ Mittels Back-calculation modellierter Ablauf der HIV-Epidemie in Deutschland in den verschiedenen Betroffenengruppen

und wie stark sich HIV in den promisk lebenden Subpopulationen der heterosexuellen Allgemeinbevölkerung ausbreiten kann.

\section{Daten der HIV- Laborberichtspflicht}

Bei der Beurteilung der aktuellen HIVEpidemie ist man, wie oben ausgeführt, wenn ansonsten keine weiteren Datenquellen zur Verfügung stehen, angewiesen auf die Daten der 1987 eingeführten HIV-Laborberichtspflicht. Diese hat aber einige gravierende Mängel - abge- sehen von der bereits erwähnten Problematik, dass Diagnose- und Infektionszeitpunkt weit auseinanderfallen können.

Durch den bewussten Verzicht auf eine namentliche Meldung sowie den Verzicht auf eine Kodierung konnten Mehrfachmeldungen nur unzureichend erkannt werden. Dies wurde bei Einführung der Laborberichtsverordnung als Problem unterschätzt. Aus Sentinelerhebungen ist bekannt, dass HIV-Infizierte im Durchschnitt etwa dreimal auf HIV-Antikörper untersucht werden [2]. Den Labors bleibt aber oftmals unbe- 
kannt, ob es sich um eine Wiederholungsuntersuchung handelt, und positive Befunde können so von ein und derselben Person mehrfach gemeldet werden. Um die daraus resultierende schlechte Datenqualität zu verbessern, wurden die Labore seit 1993 um Angabe gebeten, ob es sich - nach ihrer Kenntnis - um definitive Erstdiagnosen, definitive Wiederholungsuntersuchungen oder um Untersuchungen handelte, bei denen dem Labor nicht bekannt war, ob es sich um Erst- oder Zweituntersuchungen handelte. Die Gesamtzahl der Meldungen verteilt sich etwa zu jeweils einem Drittel auf die drei Kategorien (Erstmeldung, Wiederholungsuntersuchung, keine Angabe möglich). Punktuelle Nachfragen und Recherchen ergaben, dass sich in der Kategorie „keine Angabe möglich" zum überwiegenden Teil ebenfalls Wiederholungsuntersuchungen verbergen, die aufgrund des höheren Rechercheaufwandes aber nicht eindeutig als solche klassifiziert werden.

Das RKI geht daher davon aus, dass mit den als gesicherten HIV-Erstdiagnosen klassifizierten Meldungen der größte Teil der tatsächlichen Erstdiagnosen erfasst wird. Diese Zahlen können als Näherungswerte für die in Deutschland erfolgenden HIV-Erstdiagnosen verwendet werden, und man kann davon ausgehen, dass diese Zahlen größenordnungs- und trendmäßig die derzeit verlässlichste Zahlenbasis für die Beurteilung der aktuellen Entwicklung der HIVEpidemie in Deutschland darstellen.

Betrachtet man nun diese definitiven HIV-Erstmeldungen seit 1993, so lässt sich feststellen,

$\rightarrow$ dass die Datenqualität im Vergleich zu vorher deutlich besser ist, d.h. es liegen z.B. mehr Angaben zum vermutlichen Infektionsrisiko vor,

$\rightarrow$ und dass die Datenqualität im Laufe der Zeit sich weiter verbessert hat, so dass nunmehr für $80 \%$ der Meldungen Angaben zum Infektionsrisiko zur Verfügung stehen (Abb.3) .

Die Verteilung der Infektionsrisiken hat sich seit 1993 deutlich geändert; die meisten Infektionen werden zwar nach wie vor bei homo- und bisexuellen Männern diagnostiziert, allerdings sind die anfangs an zweiter Stelle in der Hierarchie der Betroffenengruppen stehenden i.v.-

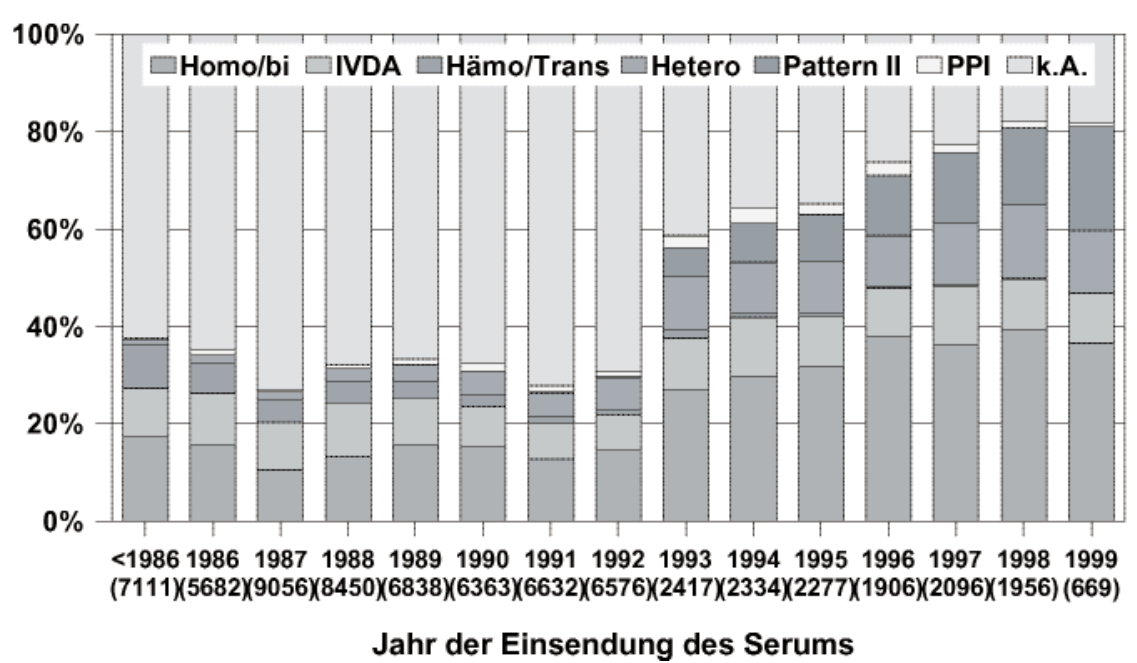

Abb.3 A Anteile der HIV-Diagnosen mit Angaben zum Infektionsrisiko und Verteilung nach Betroffenengruppen

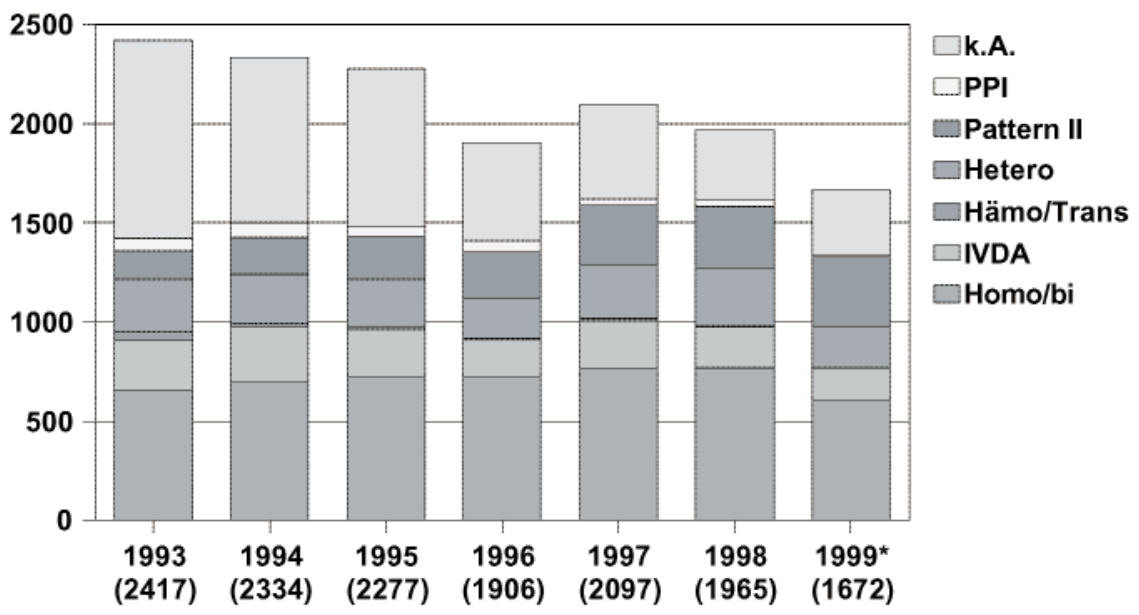

Jahr der Einsendung des Serums

Abb. 4 \ Anzahl und Verteilung der jährlich gemeldeten HIV-Neudiagnosen auf Infektionsrisiken

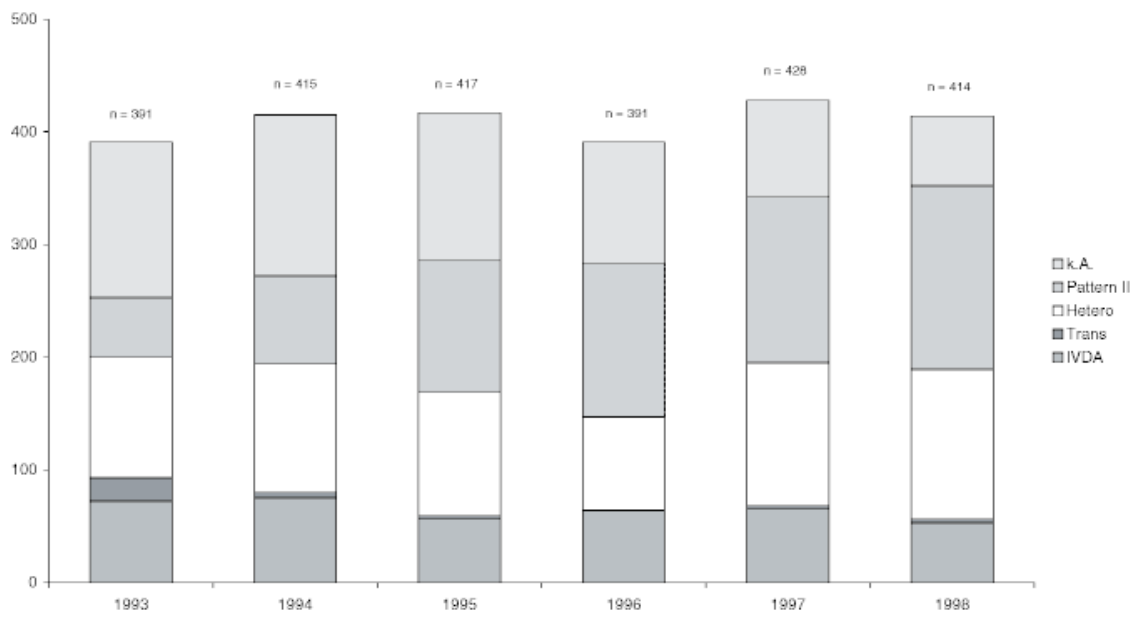

Abb.5 $\Delta$ Anzahl und Infektionsrisiken von HIV-Erstdiagnosen bei Frauen 
Drogengebraucher langsam aber sicher von der Gruppe der auf heterosexuellem Wege Infizierten und von der Gruppe der HIV-infizierten Migranten aus Hochprävalenzregionen auf den vierten Platz verdrängt worden. Mutter-Kind-Infektionen fallen mittlerweile in Deutschland glücklicherweise zahlenmäßig kaum noch ins Gewicht (Abb. 4).

Personen, die aus Ländern mit einer hohen HIV-Prävalenz stammen, wo die heterosexuelle Transmission der vorherrschende Übertragungsweg ist (sog. Pattern-II-Länder: Schwarzafrika, Karibik, Südostasien), stellen epidemiologisch eine gesonderte Gruppe mit einem hohen Infektionsrisiko dar. Bei diesen Personen wurde bisher - wenn keine anderen Angaben gemacht wurden - davon ausgegangen, dass sie die HIV-Infektion bereits in ihrem Heimatland erworben haben. Welche Rolle sexuelle Kontakte innerhalb der Gruppe der Migranten in Deutschland für Neuinfektionen spielen, ist bislang nicht untersucht worden. Bei den HIV-Meldungen nach der Laborberichtsverordnung wird das Herkunftsrisiko erst seit Mitte 1993 gesondert erfasst. Der Anteil der mit diesem Risiko erfassten gesicherten HIVErstdiagnosen ist von $5,7 \%$ für die im Jahr 1993 diagnostizierten HIV-Infektionen auf 15,9\% im Jahr 1998 deutlich angestiegen. Diese Zunahme ist sicher teilweise durch eine bessere Erfassung dieses Infektionsweges (vor allem durch vollständigere Angaben der Einsender bzw. der meldenden Laboratorien) zu erklären. Jedoch reflektiert dieser Anstieg auch die hohe Prävalenz von HIV in den Herkunftsländern dieser Personen und ist ein Hinweis dafür, dass die epidemiologische Entwicklung in anderen Ländern Deutschland nicht unberührt lässt. Insbesondere in den neuen Bundesländern, wo die Gesamtzahl der HIV-Infizierten relativ gering ist, kann der Anteil der Personen aus Hochprävalenzgebieten $30 \%$ und mehr erreichen.

Noch deutlicher werden die Verschiebungen im Spektrum der Betroffenen, wenn man nur die HIV-Infektionen bei Frauen, deren Anteil unter den HIVErstdiagnosen von 19\% im Jahr 1993 auf 23\% im Jahr 1998 zugenommen hat, betrachtet. In den achtziger Jahren bildeten aktuell oder vormals drogengebrauchende Frauen die Mehrheit der weiblichen HIV-Infizierten. Inzwischen (1998) stellen Migrantinnen (37\%) und auf he-

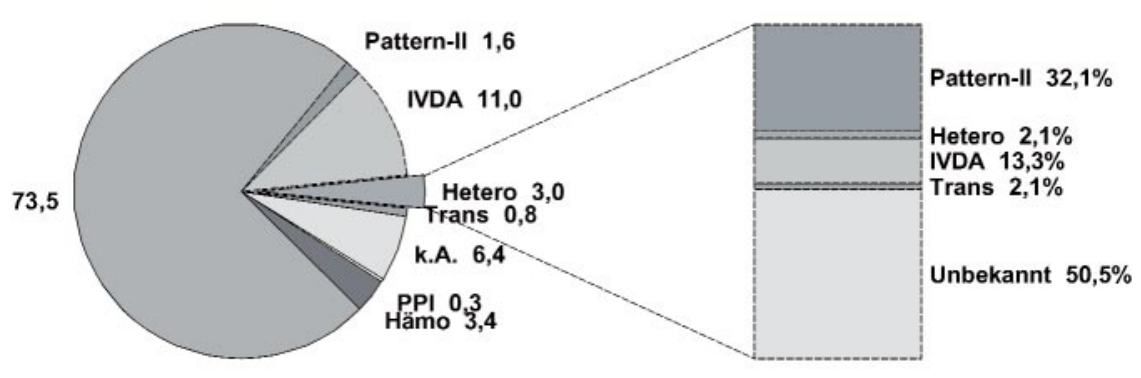

Risiko des gemeldeten Falles

Risiko der Infektionsquelle

Abb. 6 \ HIV-Infektionsrisiken bei männlichen AIDS-Patienten sowie Infektionsrisiken der vermuteten Infektionsquelle bei heterosexuell infizierten Männern

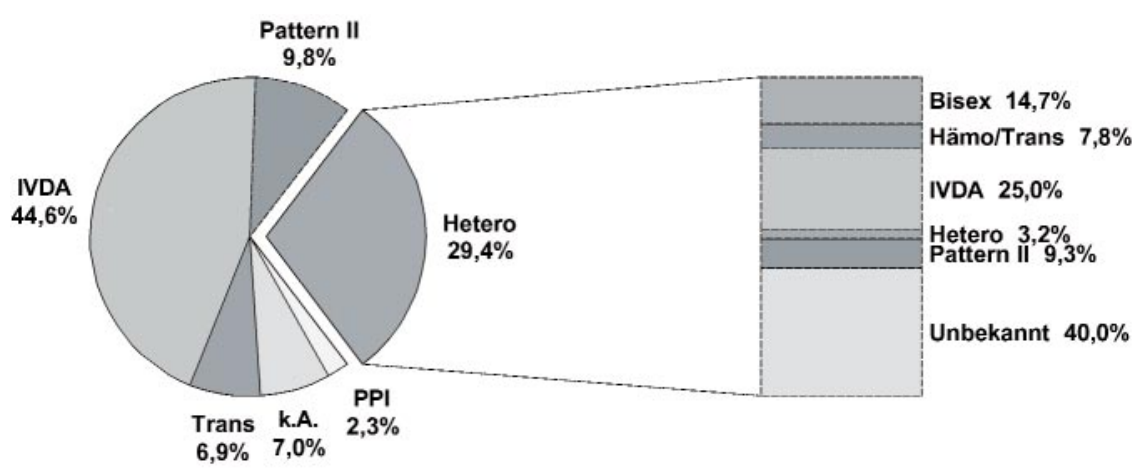

Risiko des gemeldeten Falles

Risiko der Infektionsquelle

Abb.7 $\Delta$ HIV-Infektionsrisiken bei weiblichen AIDS-Patienten sowie Infektionsrisiken der vermuteten Infektionsquelle bei heterosexuell infizierten Frauen

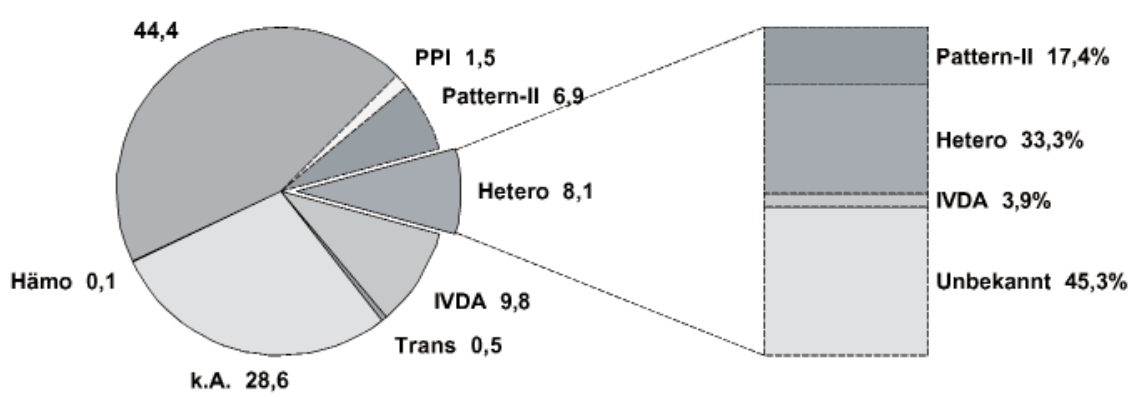

Risiko des Untersuchten

Risiko der Infektionsquelle

Abb. 8 Infektionsrisiken bei neu diagnostizierten HIV-infizierten Männern seit 1993 sowie vermutetes Infektionsrisiko der Partnerin bei heterosexuell infizierten Männern

terosexuellem Wege infizierte Frauen (30\%) die weit überwiegende Mehrheit bei den HIV-Erstdiagnosen (Abb. 5).

Deutlich wird die Verschiebung hinsichtlich der Betroffenengruppen, wenn man die Infektionsrisiken bei bislang diagnostizierten und gemeldeten AIDS-Fällen, bei denen die HIV-Infektion im Mittel etwa zehn Jahre zurückliegt, und bei den seit 1993 neu diagno- stizierten HIV-Infektionen einander, getrennt nach Männern und Frauen, gegenübergestellt. (AIDS-Fälle: Abb. 6 und 7) (HIV-Neuinfektionen seit 1993: Abb. 8 und 9).

Die bisherige Entwicklung der HIV/ AIDS-Epidemie in Deutschland lässt sich folgendermaßen zusammenfassen: Eine erste große Welle von HIVInfektionen fand in der ersten Hälfte 
der achtziger Jahre statt. Die seit 1985 eingeleiteten Präventionsbemühungen haben sicherlich entscheidend mit dazu beigetragen, dass sich die HIVAusbreitung dann in der zweiten Hälfte der achtziger Jahre deutlich verlangsamte. Es war aber unter den gegebenen Umständen nicht möglich, das etwa Ende der achtziger/ Anfang der neunziger Jahre erreichte Niveau an HIV-Neuinfektionen weiter zu reduzieren. Zweifellos erreichte Verbesserungen in einzelnen Populationen wurden durch steigende Infektionszahlen in anderen wieder ausgeglichen.

Der mit zeitlicher Verzögerung zur HIV-Ausbreitung einsetzende Anstieg der AIDS-Fälle erreichte in der ersten Hälfte der neunziger Jahre ein Plateau, das vorübergehend zu einer Art Gleichgewichtszustand führte: die Zahl der HIV-Neuinfektionen und der AIDS-Manifestationen sowie AIDS-Todesfälle hielten einander nahezu die Waage, so dass sich die Gesamtzahl der lebenden HIVInfizierten (HIV-Prävalenz) kaum noch veränderte. Dieser Gleichgewichtszustand wurde durch die Einführung und den weit verbreiteten Einsatz besser wirksamer antiretroviraler Kombinationstherapien wieder aufgehoben (Abb. 10). Seit 1995 geht die Zahl der AIDS-Neumanifestationen (AIDS-Inzidenz) und der AIDSTodesfälle (AIDS-Mortalität) durch die verbesserten Behandlungsmöglichkeiten zurück. Da die Zahl der HIV-Neuinfektionen (zumindest der Neudiagnosen) anscheinend weitgehend unverändert blieb, nimmt seit 1995 die Zahl der lebenden HIV-Infizierten und AIDSKranken allmählich zu.

Auch bei dieser Entwicklung dürfte es sich allerdings um eine vorübergehende handeln, denn bei einer steigenden Zahl von antiretroviral therapierten HIV-Infizierten entwickeln sich Resistenzen gegen die eingesetzten Medikamente, und die virologische und klinische Wirksamkeit der Therapien lässt nach. Sobald keine neuen, wirksamen Behandlungsoptionen mehr zur Verfügung stehen, muss bei diesen Personen mit einer erneuten klinischen Progredienz der HIV-Infektion gerechnet werden. Zudem besteht die Befürchtung, dass die bessere Therapierbarkeit der HIV-Infektion zu einer veränderten Ri-

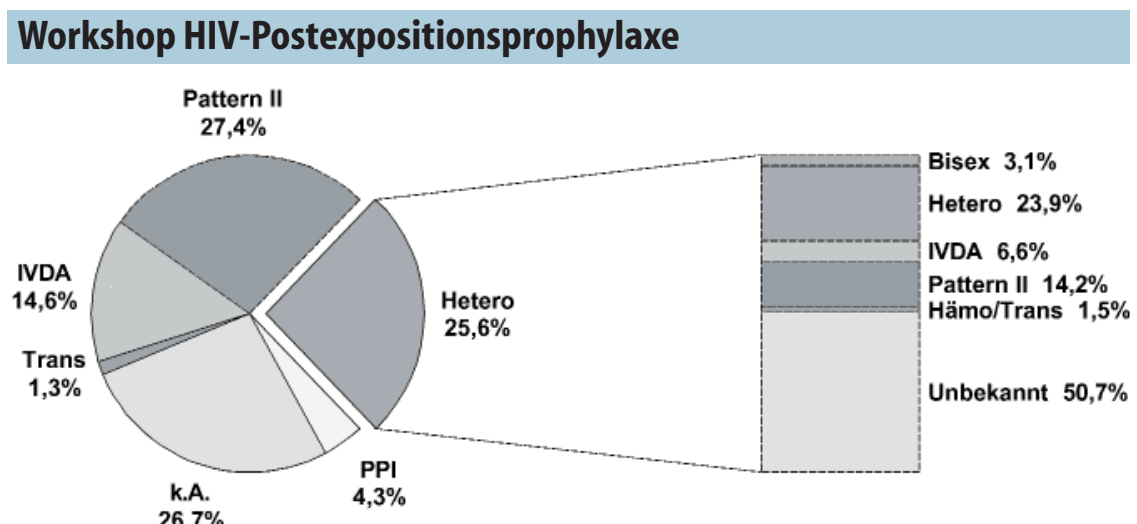

Risiko der Untersuchten

Risiko der Infektionsquelle

Abb.9 A Infektionsrisiken bei neu diagnostizierten HIV-infizierten Frauen seit 1993 sowie vermutetes Infektionsrisiko des Partners bei heterosexuell infizierten Frauen

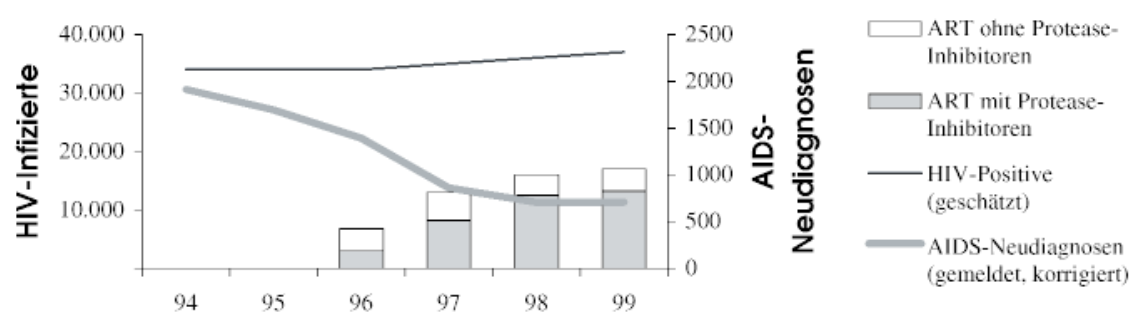

Abb. $10 \Delta$ Auswirkungen der verbesserten antiretroviralen Therapie (ART) auf Zahl der Lebenden HIV-Infizierten und der AIDS-Diagnosen

sikowahrnehmung und einer Zunahme von Risikoverhalten führen könnte, die sich ungünstigenfalls sogar in einer $\mathrm{Zu}$ nahme von HIV-Neuinfektionen niederschlagen könnte. Daten und Beobachtungen, die diese Befürchtung untermauern, werden bereits aus den USA, ansatzweise auch aus europäischen Ländern, berichtet.

Es wäre durchaus denkbar, dass eine Zunahme von Risikoverhalten zunächst durch einen Rückgang der Infektiosität unter antiretroviraler Behandlung ,neutralisiert” wird, sich positive und negative Effekte hinsichtlich der HIV-Neuinfektionsrate also eine Zeitlang gegenseitig aufheben. Für die frühzeitige Erkennung eines Trends in Richtung vermehrten Risikoverhaltens wäre daher eine bessere epidemiologische Erfassung von „Surrogatmarkern” des Risikoverhaltens, wie z.B. der Rate der Gonorrhoe-Neuinfektionen oder der Raten anderer sexuell übertragbarer Erkrankungen (STDs) mit kurzen Inkubationszeiten, hilfreich.

\section{Literatur}

1. Vanhems P, Dassa C, Lambert J, Cooper DA et al. (1999) Comprehensive classification of symptoms and signs reported among 218 patients with acute HIV-1 infection. JAIDS Journal of Acquired Immune Deficiency Syndromes 21:99-106

2. Kirschner W, Schwartländer B (1996) SentinelSurveillance von HIV und anderen sexuell übertragbaren Krankheiten. NOMOS-Verlagsgesellschaft, Baden-Baden 